\title{
Meromorphic Parabolic Starlike Functions Associated with $q$-Hypergeometric Series
}

\author{
G. Murugusundaramoorthy and T. Janani \\ School of Advanced Sciences, VIT University, Vellore 632014, India \\ Correspondence should be addressed to G. Murugusundaramoorthy; gmsmoorthy@yahoo.com
}

Received 28 February 2014; Accepted 20 March 2014; Published 7 April 2014

Academic Editors: A. Peris and S. Zhang

Copyright ( 2014 G. Murugusundaramoorthy and T. Janani. This is an open access article distributed under the Creative Commons Attribution License, which permits unrestricted use, distribution, and reproduction in any medium, provided the original work is properly cited.

We introduce a new class of meromorphic parabolic starlike functions with a fixed point defined in the punctured unit disk $\Delta^{*}:=\{z \in \mathbb{C}: 0<|z|<1\}$ involving the $q$-hypergeometric functions. We obtained coefficient inequalities, growth and distortion inequalities, and closure results for functions $f \in \mathscr{M}_{m}^{l}(\lambda, \beta, \gamma)$. We further established some results concerning convolution and the partial sums.

\section{Introduction}

Let $\xi$ be a fixed point in the unit disc $\Delta:=\{z \in \mathbb{C}:|z|<1\}$. Denote by $\mathscr{H}(\Delta)$ the class of functions which are regular and

$$
\mathscr{A}(\xi)=\left\{f \in H(\Delta): f(\xi)=f^{\prime}(\xi)-1=0\right\} .
$$

Also denote by $\mathcal{S}_{\xi}=\{f \in \mathscr{A}(\xi): f$ is univalent in $\Delta\}$, the subclass of $\mathscr{A}(\xi)$ consisting of the functions of the form

$$
f(z)=(z-\xi)+\sum_{n=2}^{\infty} a_{n}(z-\xi)^{n}
$$

which are analytic in $\Delta$. Note that $\mathcal{S}_{0}=\mathcal{S}$ is subclasses of $\mathscr{A}$ consisting of univalent functions in $\Delta$. By $\delta_{w}^{*}(\beta)$ and $\mathscr{K}_{w}(\beta)$, respectively, we mean the classes of analytic functions that satisfy the analytic conditions $\Re\left\{(z-\xi) f^{\prime}(z) / f(z)\right\}>\beta$, and $\Re\left\{1+\left((z-\xi) f^{\prime \prime}(z) / f^{\prime}(z)\right)\right\}>\beta,(z-w) \in \Delta$ for $0 \leqq \beta<1$ introduced and studied by Kanas and Ronning [1]. The class $\mathcal{S}_{\xi}^{*}(0)$ is defined by geometric property that the image of any circular arc centered at $\xi$ is starlike with respect to $f(\xi)$ and the corresponding class $\mathscr{K}_{\xi}^{*}(0)$ is defined by the property that the image of any circular arc centered at $\xi$ is convex. We observe that the definitions are somewhat similar to the ones introduced by Goodman in $[2,3]$ for uniformly starlike and convex functions, except that in this case the point $\xi$ is fixed.
In particular, $\mathscr{K}=\mathscr{K}_{0}(0)$ and $\delta_{0}^{*}=\mathcal{S}^{*}(0)$, respectively, are the well-known standard classes of convex and starlike functions.

Let $\Sigma$ denote the class of meromorphic functions $f$ of the form

$$
f(z)=\frac{1}{z}+\sum_{n=1}^{\infty} a_{n} z^{n}
$$

defined on the punctured unit disk $\Delta^{*}:=\{z \in \mathbb{C}: 0<|z|<$ $1\}$.

Denote by $\Sigma_{\xi}$ the subclass of $\Sigma$ consisting of the functions of the form

$$
f(z)=\frac{1}{z-\xi}+\sum_{n=1}^{\infty} a_{n}(z-\xi)^{n}, \quad a_{n} \geq 0 ; \quad z \neq \xi .
$$

A function $f$ of the form (4) is in the class of meromorphic starlike of order $\gamma(0 \leq \gamma<1)$ denoted by $\Sigma_{\xi}^{*}(\gamma)$, if

$$
-\Re\left(\frac{(z-\xi) f^{\prime}(z)}{f(z)}\right)>\gamma, \quad z-\xi \in \Delta:=\Delta^{*} \cup\{0\},
$$


and is in the class of meromorphic convex of order $\gamma(0 \leq \gamma<$ 1) denoted by $\Sigma_{\xi}^{K}(\gamma)$, if

$$
-\Re\left(1+\frac{(z-\xi) f^{\prime \prime}(z)}{f^{\prime}(z)}\right)>\gamma, \quad z-\xi \in \Delta:=\Delta^{*} \cup\{0\} .
$$

For functions $f(z)$ given by $(4)$ and $g(z)=(1 /(z-\xi))+$ $\sum_{n=1}^{\infty} b_{n}(z-\xi)^{n},\left(b_{n} \geq 0\right)$ we define the Hadamard product or convolution of $f$ and $g$ by

$$
(f * g)(z):=\frac{1}{z-\xi}+\sum_{n=1}^{\infty} a_{n} b_{n}(z-\xi)^{n} .
$$

More recently, Purohit and Raina [4] introduced a generalized $q$-Taylor's formula in fractional $q$-calculus and derived certain $q$-generating functions for $q$-hypergeometric functions. In this work we proceed to derive a generalized differential operator on meromorphic functions in $\Delta^{*}=\{z \epsilon$ $\mathbb{C}: 0<|z|<1\}$ involving these functions and discuss some of their properties.

For complex parameters $a_{1}, \ldots, a_{l}$ and $b_{1}, \ldots, b_{m}\left(b_{j} \neq 0\right.$, $-1, \ldots ; j=1,2, \ldots, m)$ the $q$-hypergeometric function ${ }_{l} \Psi_{m}(z)$ is defined by

$$
\begin{aligned}
{ }_{l} \Psi_{m} & \left(a_{1}, \ldots a_{l} ; b_{1}, \ldots, b_{m} ; q, z\right) \\
:= & \sum_{n=0}^{\infty} \frac{\left(a_{1}, q\right)_{n} \cdots\left(a_{l}, q\right)_{n}}{(q, q)_{n}\left(b_{1}, q\right)_{n} \cdots\left(b_{m}, q\right)_{n}} \\
& \times\left[(-1)^{n} q^{\left.\left(\begin{array}{c}
n \\
2
\end{array}\right)\right]^{1+m-l}} z^{n},\right.
\end{aligned}
$$

with $\left(\begin{array}{l}n \\ 2\end{array}\right)=n(n-1) / 2$ where $q \neq 0$ when $l>m+1(l, m \in$ $\left.\mathbb{N}_{0}=\mathbb{N} \cup\{0\} ; z \in \mathbb{U}\right)$.

The $q$-shifted factorial is defined for $a, q \in \mathbb{C}$ as a product of $n$ factors by

$$
(a ; q)_{n}= \begin{cases}1 & n=0 \\ (1-a)(1-a q) \cdots\left(1-a q^{n-1}\right) & n \in \mathbb{N},\end{cases}
$$

and in terms of basic analogue of the gamma function

$$
\left(q^{a} ; q\right)_{n}=\frac{\Gamma_{q}(a+n)(1-q)^{n}}{\Gamma_{q}(a)}, \quad n>0 .
$$

It is of interest to note that $\lim _{q \rightarrow 1^{-}}\left(\left(q^{a} ; q\right)_{n} /(1-q)^{n}\right)=(a)_{n}=$ $a(a+1) \cdots(a+n-1)$ is the familiar Pochhammer symbol and

$$
{ }_{l} \Psi_{m}\left(a_{1}, \ldots, a_{l} ; b_{1}, \ldots, b_{m} ; z\right)=\sum_{n=0}^{\infty} \frac{\left(a_{1}\right)_{n} \cdots\left(a_{l}\right)_{n}}{\left(b_{1}\right)_{n} \cdots\left(b_{m}\right)_{n}} \frac{z^{n}}{n !} .
$$

Now for $z \in \mathbb{U}, 0<|q|<1$, and $l=m+1$, the basic hypergeometric function defined in (8) takes the form

$$
\begin{aligned}
&{ }_{l} \Psi_{m}\left(a_{1} ; \ldots a_{l} ; b_{1}, \ldots, b_{m} ; q, z\right) \\
& \quad=\sum_{n=0}^{\infty} \frac{\left(a_{1}, q\right)_{n} \cdots\left(a_{l}, q\right)_{n}}{(q, q)_{n}\left(b_{1}, q\right)_{n} \cdots\left(b_{m}, q\right)_{n}} z^{n},
\end{aligned}
$$

which converges absolutely in the open unitdisk $\mathbb{U}$.
Corresponding to the function ${ }_{l} \Psi_{m}\left(a_{1} ; \ldots a_{l} ; b_{1}, \ldots, b_{m}\right.$; $q, z)$ recently for meromorphic functions $f \in \Sigma_{0}$ consisting functions of the form (3), Huda and Darus [5] introduce $q$ analogue of Liu-Srivastava operator as below:

$$
\begin{aligned}
& { }_{l} \Psi_{m}\left(a_{1} ; \ldots a_{l} ; b_{1}, \ldots, b_{m} ; q, z\right) * f(z) \\
& \quad=\frac{1}{z} \Psi_{m}\left(a_{1} ; \ldots a_{l} ; b_{1}, \ldots, b_{m} ; q, z\right) * f(z) \\
& \quad=\frac{1}{z}+\sum_{n=1}^{\infty} \frac{\left(a_{1} ; q\right)_{n+1} \cdots\left(a_{l} ; q\right)_{n+1}}{(q ; q)_{n+1}\left(b_{1} ; q\right)_{n+1} \cdots\left(b_{m}, q\right)_{n+1}} a_{n} z^{n},
\end{aligned}
$$

where $z \in \Delta^{*}:=\{z \in \mathbb{C}: 0<|z|<1\}$.

In this paper for functions $f \in \Sigma_{\xi}$ and for real parameters $a_{1}, \ldots, a_{l}$ and $b_{1}, \ldots, b_{m}\left(b_{j} \neq 0,-1, \ldots ; j=1,2, \ldots, m\right)$ we define the following new linear operator:

$$
\mathscr{I}_{m}^{l}\left(a_{1} ; \ldots a_{l} ; b_{1}, \ldots, b_{m} ; q, z-\xi\right): \Sigma_{\xi} \longrightarrow \Sigma_{\xi},
$$

as

$$
\begin{aligned}
& \mathscr{F}_{m}^{l}\left(a_{1} ; \ldots a_{l} ; b_{1}, \ldots, b_{m} ; q, z-\xi\right) \\
& \quad=\frac{1}{z-\xi} \Psi_{m}\left(a_{1} ; \ldots a_{l} ; b_{1}, \ldots, b_{m} ; q, z-\xi\right) \\
& \mathscr{F}_{m}^{l}\left[a_{l}, q\right]=\frac{1}{z-\xi}+\sum_{n=1}^{\infty} \Upsilon_{n}^{l, m}\left[a_{1}, q\right](z-\xi)^{n}
\end{aligned}
$$

where

$$
\begin{aligned}
Y_{n}^{l, m} & {\left[a_{1}, q\right] } \\
& =\frac{\left(a_{1} ; q\right)_{n+1} \cdots\left(a_{l} ; q\right)_{n+1}}{(q ; q)_{n+1}\left(b_{1} ; q\right)_{n+1} \cdots\left(b_{m}, q\right)_{n+1}} .
\end{aligned}
$$

Throughout our study for $f \in \Sigma_{\xi}$, we let

$$
\begin{aligned}
\mathscr{F}_{m}^{l} f(z) & =\mathcal{F}_{m}^{l}\left[a_{l}, q\right] * f(z) \\
& =\frac{1}{z-\xi}+\sum_{n=1}^{\infty} \Upsilon_{m}^{l}(n) a_{n}(z-\xi)^{n}, \\
\Upsilon_{m}^{l}(n) & =\Upsilon_{n}^{l, m}\left[a_{1}, q\right] \\
& =\frac{\left(a_{1} ; q\right)_{n+1} \cdots\left(a_{l} ; q\right)_{n+1}}{(q ; q)_{n+1}\left(b_{1} ; q\right)_{n+1} \cdots\left(b_{m}, q\right)_{n+1}},
\end{aligned}
$$

unless otherwise stated.

Motivated by earlier works on meromorphic functions by function theorists (see [6-14]), we define the following new subclass of functions in $\Sigma_{\xi}$ by making use of the generalized operator $\mathscr{I}_{m}^{l}$. 
For $0 \leq \gamma<1$ and $0 \leq \lambda<1 / 2$, we let $\mathscr{M}_{m}^{l}(\lambda, \beta, \gamma)$ denote a subclass of $\Sigma_{\xi}$ consisting functions of the form (4) satisfying the condition that

$$
\begin{aligned}
& -\Re\left(\frac{(z-\xi)\left(\mathscr{J}_{m}^{l} f(z)\right)^{\prime}+\lambda(z-\xi)^{2}\left(\mathscr{F}_{m}^{l} f(z)\right)^{\prime \prime}}{(1-\lambda) \mathscr{F}_{m}^{l} f(z)+\lambda(z-\xi)\left(\mathscr{F}_{m}^{l} f(z)\right)^{\prime}}\right) \\
& >\beta\left|\frac{(z-\xi)\left(\mathscr{F}_{m}^{l} f(z)\right)^{\prime}+\lambda(z-\xi)^{2}\left(\mathscr{F}_{m}^{l} f(z)\right)^{\prime \prime}}{(1-\lambda) \mathscr{I}_{m}^{l} f(z)+\lambda(z-\xi)\left(\mathscr{F}_{m}^{l} f(z)\right)^{\prime}}+1\right| \\
& \quad+\gamma,
\end{aligned}
$$

where $\mathscr{F}_{m}^{l} f$ is given by (17).

Further, shortly we can state this condition by

$$
-\Re\left(\frac{(z-\xi) G^{\prime}(z)}{G(z)}\right)>\beta\left|\frac{(z-\xi) G^{\prime}(z)}{G(z)}+1\right|+\gamma,
$$

where

$$
\begin{array}{r}
G(z)=(1-\lambda) \mathscr{F}_{m}^{l} f(z)+\lambda(z-\xi)\left(\mathscr{F}_{m}^{l} f(z)\right)^{\prime} \\
=\frac{1-2 \lambda}{z-\xi}+\sum_{n=1}^{\infty}(n \lambda-\lambda+1) \Upsilon_{m}^{l}(n) a_{n}(z-\xi)^{n}, \\
a_{n} \geq 0 .
\end{array}
$$

It is of interest to note that, on specializing the parameters $\lambda, \beta$ and $l, m$, we can define various new subclasses of $\Sigma_{\xi}$. We illustrate two important subclasses in the following examples.

Example 1. For $\lambda=0$, we let $\mathscr{M}_{m}^{l}(0, \beta, \gamma)=\mathscr{M}_{m}^{l}(\beta, \gamma)$ denote a subclass of $\Sigma_{\xi}$ consisting functions of the form (4) satisfying the condition that

$$
\begin{aligned}
- & \Re\left(\frac{(z-\xi)\left(\mathscr{I}_{m}^{l} f(z)\right)^{\prime}}{\mathscr{I}_{m}^{l} f(z)}\right) \\
& >\beta\left|\frac{(z-\xi)\left(\mathscr{I}_{m}^{l} f(z)\right)^{\prime}}{\mathscr{I}_{m}^{l} f(z)}+1\right|+\gamma,
\end{aligned}
$$

where $\mathscr{F}_{m}^{l} f(z)$ is given by (17).

Example 2. For $\lambda=0, \beta=0$ we let $\mathscr{M}_{m}^{l}(0,0, \gamma)=\mathscr{M}_{m}^{l}(\gamma)$ denote a subclass of $\Sigma_{\xi}$ consisting functions of the form (4) satisfying the condition that

$$
-\Re\left(\frac{(z-\xi)\left(\mathscr{F}_{m}^{l} f(z)\right)^{\prime}}{\mathscr{F}_{m}^{l} f(z)}\right)>\gamma,
$$

where $\mathscr{F}_{m}^{l} f(z)$ is given by (17).

In this paper, we obtain the coefficient inequalities, growth and distortion inequalities, and closure results for the function class $\mathscr{M}_{m}^{l}(\lambda, \beta, \gamma)$. Properties of certain integral operator and convolution properties of the new class $\mathscr{M}_{m}^{l}(\lambda, \beta, \gamma)$ are also discussed.

\section{Coefficients Inequalities}

In order to obtain the necessary and sufficient condition for a function, $f \in \mathscr{M}_{m}^{l}(\lambda, \beta, \gamma)$, we recall the following lemmas.

Lemma 3. If $\gamma$ is a real number and $w$ is a complex number, then $\mathfrak{R}(w) \geq \gamma \Leftrightarrow|w+(1-\gamma)|-|w-(1+\gamma)| \geq 0$.

Lemma 4. If $w$ is a complex number and $\gamma, k$ are real numbers, then

$$
\begin{array}{r}
\mathfrak{R}(w) \geq k|w-1|+\gamma \Longleftrightarrow \mathfrak{R}\left\{w\left(1+k e^{i \theta}\right)-k e^{i \theta}\right\} \geq \gamma, \\
-\pi \leq \theta \leq \pi .
\end{array}
$$

Analogous to the lemma proved by Dziok et al. [8], we state the following lemma without proof.

Lemma 5. Suppose that $\gamma \in[0,1), r \in(0,1]$, and the function $H \in \Sigma_{\xi}(\gamma)$ is of the form $H(z)=(1 /(z-\xi))+\sum_{n=1}^{\infty} b_{n}(z-\xi)^{n}$, $0<|z-\xi|<r<1$, with $b_{n} \geq 0$, then

$$
\sum_{n=1}^{\infty}(n+\gamma) b_{n} r^{n+1} \leq 1-\gamma
$$

Theorem 6. Let $f \in \Sigma_{\xi}$ be given by (4). Then $f \in M_{m}^{l}(\lambda, \beta, \gamma)$ if and only if

$$
\begin{aligned}
& \sum_{n=1}^{\infty}[n(1+\beta)+(\gamma+\beta)](1+n \lambda-\lambda) \Upsilon_{m}^{l}(n) a_{n} \\
& \leq(1-2 \lambda)(1-\gamma) .
\end{aligned}
$$

Proof. If $f \in \mathscr{M}_{m}^{l}(\lambda, \beta, \gamma)$, then by (20) we have

$$
-\mathfrak{R}\left(\frac{(z-\xi) G^{\prime}(z)}{G(z)}\right)>\beta\left|\frac{(z-\xi) G^{\prime}(z)}{G(z)}+1\right|+\gamma .
$$

Making use of Lemma 4,

$$
-\Re\left(\frac{(z-\xi)\left(1+\beta e^{i \theta}\right) G^{\prime}(z)+\beta e^{i \theta} G(z)}{G(z)}\right)>\gamma,
$$

where $G(z)$ is given by (21). Substituting $G(z), G^{\prime}(z)$ and letting $|z-\xi|<r \rightarrow 1^{-}$, we have

$$
\begin{aligned}
& \left\{\left((1-2 \lambda)(1-\gamma)-\sum_{n=1}^{\infty}[n(1+\beta)+(\gamma+\beta)]\right.\right. \\
& \left.\quad \times(1+n \lambda-\lambda) \Upsilon_{m}^{l}(n) a_{n}\right) \\
& \left.\quad \times\left((1-2 \lambda)-\sum_{n=1}^{\infty}(1+n \lambda-\lambda) \Upsilon_{m}^{l}(n) a_{n}\right)^{-1}\right\}>0 .
\end{aligned}
$$

This shows that (26) holds. 
Conversely, assume that (26) holds. Since $-\mathfrak{R}(w)>\gamma$, if and only if $|w+1|<|w-(1-2 \gamma)|$, it is sufficient to show that

$$
\begin{array}{r}
\left|\frac{w+1}{w-(1-2 \gamma)}\right|<1, \quad|w-(1-2 \gamma)| \neq 0 \\
\text { for }|z-\xi|<r \leq 1, \quad(z-\xi) \in \Delta .
\end{array}
$$

Using (26) and taking $w(z)=\left((z-\xi)\left(1+\beta e^{i \theta}\right) G^{\prime}(z)+\right.$ $\left.\beta e^{i \theta} G(z)\right) / G(z)$, we get

$$
\begin{aligned}
& \left|\frac{w+1}{w-(1-2 \gamma)}\right| \\
& \leq\left(\left(\sum_{n=1}^{\infty}(1+n \lambda-\lambda)[(n+1)(1+\beta)] \Upsilon_{m}^{l}(n) a_{n}\right)\right. \\
& \quad \times\left(2(1-\gamma)(1-2 \lambda)-\sum_{n=1}^{\infty}(1+n \lambda-\lambda)\right. \\
& \left.\left.\quad \times[n(1+\beta)+(\beta+2 \gamma-1)] \Upsilon_{m}^{l}(n) a_{n}\right)^{-1}\right)
\end{aligned}
$$

$\leq 1$.

Thus we have $f \in \mathscr{M}_{m}^{l}(\lambda, \beta, \gamma)$.

For the sake of brevity throughout this paper we let

$$
\begin{aligned}
& d_{n}(\lambda, \beta, \gamma)=[n(1+\beta)+(\gamma+\beta)](1+n \lambda-\lambda), \\
& d_{1}(\lambda, \beta, \gamma)=(1+\gamma+2 \beta),
\end{aligned}
$$

unless otherwise stated.

Our next result gives the coefficient estimates for functions in $\mathscr{M}_{m}^{l}(\lambda, \beta, \gamma)$.

Theorem 7. If $f \in \mathscr{M}_{m}^{l}(\lambda, \beta, \gamma)$, then

$$
a_{n} \leq \frac{(1-\gamma)(1-2 \lambda)}{d_{n}(\lambda, \beta, \gamma) Y_{m}^{l}(n)}, \quad n=1,2,3, \ldots
$$

The result is sharp for the functions $f_{n}(z)$ given by

$$
\begin{array}{r}
f_{n}(z)=\frac{1}{z-\xi}+\frac{1-\gamma}{d_{n}(\lambda, \beta, \gamma) \Upsilon_{m}^{l}(n)}(z-\xi)^{n}, \\
n=1,2,3, \ldots .
\end{array}
$$

Proof. If $f \in \mathscr{M}_{m}^{l}(\lambda, \beta, \gamma)$, then we have, for each $n$,

$$
\begin{aligned}
d_{n}(\lambda, \beta, \gamma) \Upsilon_{m}^{l}(n) a_{n} & \leq \sum_{n=1}^{\infty} d_{n}(\lambda, \beta, \gamma) \Upsilon_{m}^{l}(n) a_{n} \\
& \leq(1-\gamma)(1-2 \lambda) .
\end{aligned}
$$

Therefore we have

$$
a_{n} \leq \frac{(1-\gamma)(1-2 \lambda)}{d_{n}(\lambda, \beta, \gamma) \Upsilon_{m}^{l}(n)} .
$$

Since

$$
f_{n}(z)=\frac{1}{z-\xi}+\frac{(1-\gamma)(1-2 \lambda)}{d_{n}(\lambda, \beta, \gamma) \Upsilon_{m}^{l}(n)}(z-\xi)^{n}
$$

satisfies the conditions of Theorem $6, f_{n}(z) \in \mathscr{M}_{m}^{l}(\lambda, \beta, \gamma)$ and the equality is attained for this function.

Theorem 8. Suppose that there exists a positive number $v$ :

$$
\nu=\inf _{n \in \mathbb{N}}\left\{d_{n}(\lambda, \beta, \gamma) \Upsilon_{m}^{l}(n)\right\} .
$$

If $f \in \mathscr{M}_{m}^{l}(\lambda, \beta, \gamma)$, then

$$
\begin{aligned}
\mid \frac{1}{r}- & \frac{(1-\gamma)(1-2 \lambda)}{\nu} r \mid \\
\leq & |f(z)| \leq \frac{1}{r} \\
& +\frac{(1-\gamma)(1-2 \lambda)}{\nu} r, \quad(|z-\xi|=r), \\
\mid \frac{1}{r^{2}}- & \frac{(1-\gamma)(1-2 \lambda)}{\nu} \mid \\
\leq & \left|f^{\prime}(z)\right| \leq \frac{1}{r^{2}} \\
& +\frac{(1-\gamma)(1-2 \lambda)}{v} \quad(|z-\xi|=r) .
\end{aligned}
$$

If $\nu=d_{1}(\lambda, \beta, \gamma) \Upsilon_{m}^{l}(1)=(1+\gamma+2 \beta) \Upsilon_{m}^{l}(1)$, then the result is sharp for

$$
f(z)=\frac{1}{z-\xi}+\frac{(1-\gamma)(1-2 \lambda)}{(1+\gamma+2 \beta) r^{2} \Upsilon_{m}^{l}(1)}(z-\xi) .
$$

Proof. Let $f \in \sum_{\xi}$ and be given by (4)

$$
|f(z)| \leq \frac{1}{r}+\sum_{n=1}^{\infty} a_{n} r^{n} \leq \frac{1}{r}+r \sum_{n=1}^{\infty} a_{n} .
$$

Since $f \in \mathscr{M}_{m}^{l}(\lambda, \beta, \gamma)$, and by Theorem 6 ,

$$
\sum_{n=1}^{\infty} a_{n} \leq \frac{(1-\gamma)(1-2 \lambda)}{\nu} .
$$

Using this, we have

$$
|f(z)| \leq \frac{1}{r}+\frac{(1-\gamma)(1-2 \lambda)}{\nu} r .
$$

Similarly

$$
|f(z)| \geq\left|\frac{1}{r}-\frac{(1-\gamma)(1-2 \lambda)}{\nu} r\right| .
$$

The result is sharp for function (40) with

$$
\nu=d_{1}(\lambda, \beta, \gamma) \Upsilon_{m}^{l}(1)=(1+\gamma+2 \beta) \Upsilon_{m}^{l}(1) .
$$

Similarly we can prove the otherinequality $\left|f^{\prime}(z)\right|$. 


\section{Order of Starlikeness}

In the following theorem we obtain the order of starlikeness for the class $\mathscr{M}_{m}^{l}(\lambda, \beta, \gamma)$. We say that $f$ given by (4) is meromorphically starlike of order $\rho,(0 \leq \rho<1)$, in $|z-\xi|<r$ when it satisfies condition (5) in $|z-\xi|<r$.

Theorem 9. Let the function $f$ given by (4) be in the class $\mathscr{M}_{m}^{l}(\lambda, \beta, \gamma)$. Then, if there exists

$$
r=r_{1}(\lambda, \gamma, \rho)=\inf _{n \geq 1}\left[\frac{(1-\rho) d_{n}(\lambda, \beta, \gamma) \Upsilon_{m}^{l}(n)}{(n+\rho)(1-\gamma)(1-2 \lambda)}\right]^{1 /(n+1)}
$$

and it is positive, then $f$ is meromorphically starlike of order $\rho$ in $|z-\xi|<r \leq r_{1}(\lambda, \gamma, \rho)$.

Proof. Let the function $f \in \mathscr{M}_{m}^{l}(\lambda, \beta, \gamma)$ be of the form (4). If $0<r \leq r_{1}(\lambda, \gamma, \rho)$, then by $(46)$

$$
r^{n+1} \leq \frac{(1-\rho) d_{n}(\lambda, \beta, \gamma) \Upsilon_{m}^{l}(n)}{(n+\rho)(1-\gamma)(1-2 \lambda)},
$$

for all $n \in \mathbb{N}$. From (47) we get

$$
\frac{n+\rho}{1-\rho} r^{n+1} \leq \frac{d_{n}(\lambda, \beta, \gamma) \Upsilon_{m}^{l}(n)}{(1-\gamma)(1-2 \lambda)}
$$

for all $n \in \mathbb{N}$, and thus

$$
\sum_{n=1}^{\infty} \frac{n+\rho}{1-\rho} a_{n} r^{n+1} \leq \sum_{n=1}^{\infty} \frac{d_{n}(\lambda, \beta, \gamma) \Upsilon_{m}^{l}(n)}{(1-\gamma)(1-2 \lambda)} a_{n} \leq 1,
$$

because of (26). Hence, from (49) and (25), $f$ is meromorphically starlike of order $\rho$ in $|z-\xi|<r \leq r_{1}(\lambda, \gamma, \rho)=r$.

Suppose that there exists a number $\widetilde{r}, \widetilde{r}>r_{1}(\lambda, \gamma, \rho)$, such that each $f \in \mathscr{M}_{m}^{l}(\lambda, \beta, \gamma)$ is meromorphically starlike of order $\rho$ in $|z-\xi|<\widetilde{r} \leq 1$. The function

$$
f(z)=\frac{1}{z-\xi}+\frac{(1-\gamma)(1-2 \lambda)}{d_{n}(\lambda, \beta, \gamma) \Upsilon_{m}^{l}(n)}(z-\xi)^{n}
$$

is in the class $\mathscr{M}_{m}^{l}(\lambda, \beta, \gamma)$; thus it should satisfy (25) with $\tilde{r}$ :

$$
\sum_{n=1}^{\infty}(n+\rho) a_{n} \widetilde{r}^{n+1} \leq 1-\rho,
$$

while the left-hand side of (51) becomes

$$
\begin{aligned}
(n & +\rho) \frac{(1-\gamma)(1-2 \lambda)}{d_{n}(\lambda, \beta, \gamma) \Upsilon_{m}^{l}(n)} \widetilde{r}^{n+1} \\
& >(n+\rho) \frac{(1-\gamma)(1-2 \lambda)}{d_{n}(\lambda, \beta, \gamma) \Upsilon_{m}^{l}(n)} \frac{(1-\rho) d_{n}(\lambda, \beta, \gamma) \Upsilon_{m}^{l}(n)}{(n+\rho)(1-\gamma)(1-2 \lambda)} \\
& =1-\rho,
\end{aligned}
$$

which contradicts (51). Therefore the number $r_{1}(\lambda, \gamma, \rho)$ in Theorem 9 cannot be replaced with a greater number. This means that $r_{1}(\lambda, \gamma, \rho)$ is called radius of meromorphically starlikeness of order $\rho$ for the class $\mathscr{M}_{m}^{l}(\lambda, \beta, \gamma)$.

\section{Results Involving Modified Hadamard Products}

For functions

$$
f_{j}(z)=\frac{1}{z-\xi}+\sum_{n=1}^{\infty} a_{n, j}(z-\xi)^{n}, \quad a_{n, j} \geq 0,
$$

we define the Hadamard product or convolution of $f_{1}$ and $f_{2}$ by

$$
\left(f_{1} * f_{2}\right)(z):=\frac{1}{z-\xi}+\sum_{n=1}^{\infty} a_{n, 1} a_{n, 2}(z-\xi)^{n} .
$$

Let

$$
\Psi(n, \lambda)=\frac{(n \lambda-\lambda+1)}{(1-2 \lambda)} \Upsilon_{m}^{l}(n)
$$

Theorem 10. For functions $f_{j}(j=1,2)$ defined by (53), let $f_{1} \in \mathscr{M}_{m}^{l}(\lambda, \beta, \gamma)$ and $f_{2} \in \mathscr{M}_{m}^{l}(\lambda, \beta, \delta)$. Then $f_{1} * f_{2} \in$ $\mathscr{M}_{m}^{l}(\lambda, \beta, \eta)$ where

$\eta$

$$
=1-\frac{(1-\gamma)(1-\delta)(3+\beta)}{(1+\gamma+2 \beta)(1+\delta+2 \beta) \Psi(1, \lambda)-2(1-\gamma)(1-\delta)},
$$

and $\Psi(1, \lambda)=Y_{m}^{l}(1) /(1-2 \lambda)$. The results are the best possible for

$$
\begin{aligned}
& f_{1}(z)=\frac{1}{z-\xi}+\frac{1-\gamma}{(1+\gamma+2 \beta) \Psi(1, \lambda)}(z-\xi), \\
& f_{2}(z)=\frac{1}{z-\xi}+\frac{1-\delta}{(1+\delta+2 \beta) \Psi(1, \lambda)}(z-\xi),
\end{aligned}
$$

where $\Psi(1, \lambda)=\Upsilon_{m}^{l}(1) /(1-2 \lambda)$.

Proof. In view of Theorem 6, it suffices to prove that

$$
\sum_{n=1}^{\infty} \frac{[n(1+\beta)+(\eta+\beta)]}{(1-\eta)} \Psi(n, \lambda) a_{n, 1} a_{n, 2} \leq 1,
$$

where $\eta$ is defined by (56) under the hypothesis. It follows from (26) and the Cauchy-Schwarz inequality that

$$
\begin{gathered}
\sum_{n=1}^{\infty} \frac{[n(1+\beta)+(\gamma+\beta)]^{1 / 2}[n(1+\beta)+(\delta+\beta)]^{1 / 2}}{\sqrt{(1-\gamma)(1-\delta)}} \\
\times \Psi(n, \lambda) \sqrt{a_{n, 1} a_{n, 2}} \leq 1 .
\end{gathered}
$$


Thus we need to find the largest $\eta$ such that

$$
\begin{aligned}
& \sum_{n=1}^{\infty} \frac{[n(1+\beta)+(\eta+\beta)]}{(1-\eta)} \Psi(n, \lambda) a_{n, 1} a_{n, 2} \\
& \leq \sum_{n=1}^{\infty} \frac{[n(1+\beta)+(\gamma+\beta)]^{1 / 2}[n(1+\beta)+(\delta+\beta)]^{1 / 2}}{\sqrt{(1-\gamma)(1-\delta)}} \\
& \quad \times \Psi(n, \lambda) \sqrt{a_{n, 1} a_{n, 2}} \\
& \leq 1
\end{aligned}
$$
that

By virtue of (59) it is sufficient to find the largest $\eta$, such

$$
\begin{gathered}
\frac{\sqrt{(1-\gamma)(1-\delta)}}{[n(1+\beta)+(\gamma+\beta)]^{1 / 2}[n(1+\beta)+(\delta+\beta)]^{1 / 2} \Psi(n, \lambda)} \\
\leq \frac{[n(1+\beta)+(\gamma+\beta)]^{1 / 2}[n(1+\beta)+(\delta+\beta)]^{1 / 2}}{\sqrt{(1-\gamma)(1-\delta)}} \\
\times \frac{1-\eta}{[n(1+\beta)+(\eta+\beta)]},
\end{gathered}
$$

which yields

$$
\begin{aligned}
\eta \leq 1-( & ((1-\gamma)(1-\delta)(2 n+1+\beta)) \\
\times & ([n(1+\beta)+(\gamma+\beta)][n(1+\beta)+(\delta+\beta)] \\
& \left.\times \Psi(n, \lambda)-(1-\gamma)(1-\delta)(n+1))^{-1}\right),
\end{aligned}
$$

for $n \geq 1$ where $\Psi(n, \lambda)$ is given by $(55)$ and, since $\Psi(n, \lambda)$ is a decreasing function of $n(n \geq 1)$, we have

$$
\eta=1
$$

$$
-\frac{(1-\gamma)(1-\delta)(3+\beta)}{(1+\gamma+2 \beta)(1+\delta+2 \beta) \Psi(1, \lambda)-2(1-\gamma)(1-\delta)},
$$

and $\Psi(1, \lambda)=Y_{m}^{l}(1) /(1-2 \lambda)$, which completes the proof.

Theorem 11. Let the functions $f_{j},(j=1,2)$, defined by (53) be in the class $\mathscr{M}_{m}^{l}(\lambda, \beta, \gamma)$. Then $\left(f_{1} * f_{2}\right)(z) \in \mathscr{M}_{m}^{l}(\lambda, \beta, \eta)$ where

$$
\eta=1-\frac{(1-\gamma)^{2}(3+\beta)}{(1+\gamma+2 \beta)^{2} \Psi(1, \lambda)-2(1-\gamma)^{2}}
$$

with $\Psi(1, \lambda)=\Upsilon_{m}^{l}(1) /(1-2 \lambda)$.

Proof. By taking $\delta=\gamma$ in the above theorem, the results follow.
For functions in the class $\mathscr{M}_{m}^{l}(\lambda, \beta, \gamma)$, we can prove the following inclusion property.

Theorem 12. Let the functions $f_{j}(j=1,2)$ defined by (53) be in the class $\mathscr{M}_{m}^{l}(\lambda, \beta, \gamma)$. Then the function $h$, defined by

$$
h(z)=\frac{1}{z-\xi}+\sum_{n=1}^{\infty}\left(a_{n, 1}^{2}+a_{n, 2}^{2}\right)(z-\xi)^{n}
$$

is in the class $\mathscr{M}_{m}^{l}(\lambda, \beta, \delta)$ where

$$
\delta \leq 1-\frac{4(1-\gamma)^{2}(1+\beta)}{[1+\gamma+2 \beta]^{2} \Psi(1, \lambda)+2(1-\gamma)^{2}},
$$

and $\Psi(1, \lambda)=\Upsilon_{m}^{l}(1) /(1-2 \lambda)$.

Proof. In view of Theorem 6, it is sufficient to prove that

$$
\sum_{n=2}^{\infty} \Psi(n, \lambda) \frac{[n(1+\beta)+(\delta+\beta)]}{(1-\delta)}\left(a_{n, 1}^{2}+a_{n, 2}^{2}\right) \leq 1,
$$

where $f_{j} \in \mathscr{M}_{m}^{l}(\lambda, \beta, \gamma)(j=1,2)$; we find from (53) and Theorem 6 that

$$
\begin{gathered}
\sum_{n=1}^{\infty}\left[\Psi(n, \lambda) \frac{[n(1+\beta)+(\gamma+\beta)]}{1-\gamma}\right]^{2} a_{n, j}^{2} \\
\leq \sum_{n=1}^{\infty}\left[\Psi(n, \lambda) \frac{[n(1+\beta)+(\gamma+\beta)]}{1-\gamma} a_{n, j}\right]^{2} \leq 1,
\end{gathered}
$$

which would yield

$$
\sum_{n=2}^{\infty} \frac{1}{2}\left[\Psi(n, \lambda) \frac{[n(1+\beta)+(\gamma+\beta)]}{1-\gamma}\right]^{2}\left(a_{n, 1}^{2}+a_{n, 2}^{2}\right) \leq 1
$$

On comparing (67) and (69) it can be seen that inequality (66) will be satisfied if

$$
\begin{aligned}
\Psi(n, \lambda) & \frac{[n(1+\beta)+(\delta+\beta)]}{1-\delta}\left(a_{n, 1}^{2}+a_{n, 2}^{2}\right) \\
\leq & \frac{1}{2}\left[\Psi(n, \lambda) \frac{[n(1+\beta)+(\gamma+\beta)]}{1-\gamma}\right]^{2} \\
& \times\left(a_{n, 1}^{2}+a_{n, 2}^{2}\right) .
\end{aligned}
$$

That is, if

$$
\delta \leq 1-\frac{2(1-\gamma)^{2}[(n+1)(1+\beta)]}{[n(1+\beta)+(\gamma+\beta)]^{2} \Psi(n, \lambda)+2(1-\gamma)^{2}},
$$

where $\Psi(n, \lambda)$ is given by (55) and $\Psi(n, \lambda)$ is a decreasing function of $n \quad(n \geq 1)$, we get (66), which completes the proof. 


\section{Closure Theorems}

We state the following closure theorems for $f \in \mathscr{M}_{m}^{l}(\lambda, \beta, \gamma)$ without proof (see [8-10]).

Theorem 13. Let the function $f_{k}(z)=(1 /(z-\xi))+\sum_{n=1}^{\infty} a_{n, k}$ $(z-\xi)^{n}$ be in the class $\mathscr{M}_{m}^{l}(\lambda, \beta, \gamma)$ for every $k=1,2, \ldots, m$. Then the function $f$ defined by

$$
f(z)=\frac{1}{z-\xi}+\sum_{n=1}^{\infty} a_{n, k}(z-\xi)^{n}, \quad\left(a_{n, k} \geq 0\right)
$$

belongs to the class $\mathscr{M}_{m}^{l}(\lambda, \beta, \gamma)$, where $a_{n, k}=(1 / m) \sum_{k=1}^{m} a_{n, k}$, $(n=1,2, \ldots)$.

Theorem 14. Let $f_{0}(z)=1 /(z-\xi)$ and $f_{n}(z)=(1 /(z-\xi))+$ $\left((1-\gamma)(1-2 \lambda) / d_{n}(\lambda, \beta, \gamma) \Upsilon_{m}^{l}(n)\right)(z-\xi)^{n}$ for $n=1,2, \ldots .$. Then $f \in \mathscr{M}_{m}^{l}(\lambda, \beta, \gamma)$ if and only if $f$ can be expressed in the form $f(z)=\sum_{n=0}^{\infty} \eta_{n} f_{n}(z)$ where $\eta_{n} \geq 0$ and $\sum_{n=0}^{\infty} \eta_{n}=1$.

Theorem 15. The class $\mathscr{M}_{m}^{l}(\lambda, \beta, \gamma)$ is closed under convex linear combination.

Now, we prove that the class is $\mathscr{M}_{m}^{l}(\lambda, \beta, \gamma)$ closed under integral transforms.

Theorem 16. Let the function $f(z)$ given by (4) be in $\mathscr{M}_{m}^{l}(\lambda, \beta, \gamma)$. Then the integral operator

$$
F(z)=c \int_{0}^{1} u^{c} f(u z) d u \quad(0<u \leq 1,0<c<\infty)
$$

is in $\mathscr{M}_{m}^{l}(\lambda, \beta, \delta)$, where

$$
\begin{aligned}
\delta \leq( & n^{2}(1+\beta)+n[(\gamma+\beta)+(1+\beta)(1+c \gamma)] \\
+ & (c+1)(\gamma+\beta)+c \beta(1-\gamma)) \\
\times & \left(n^{2}(1+\beta)+n[(\gamma+\beta)+(1+c)(1+\beta)]\right. \\
& +(1+c)(\gamma+\beta)+c(1-\gamma))^{-1} .
\end{aligned}
$$

The result is sharp for the function $f(z)=(1 /(z-\xi))+$ $\left((1-\gamma)(1-2 \lambda) /(1+\gamma+2 \beta) \Upsilon_{m}^{l}(1)\right)(z-\xi)$.

Proof. Let $f(z) \in \mathscr{M}_{m}^{l}(\lambda, \beta, \gamma)$. Then

$$
F(z)=c \int_{0}^{1} u^{c} f(u z) d u=\frac{1}{z-w}+\sum_{n=1}^{\infty} \frac{c}{c+n+1} a_{n}(z-\xi)^{n} .
$$

It is sufficient to show that

$$
\sum_{n=1}^{\infty} \frac{c d_{n}(\lambda, \beta, \delta) \Upsilon_{m}^{l}(n)}{(c+n+1)(1-\delta)} a_{n} \leq 1 .
$$

Since $f \in \mathscr{M}_{m}^{l}(\lambda, \beta, \gamma)$, we have

$$
\sum_{n=1}^{\infty} \frac{d_{n}(\lambda, \beta, \gamma) \Upsilon_{m}^{l}(n)}{(1-\gamma)(1-2 \lambda)} a_{n} \leq 1 .
$$

Note that (76) is satisfied if

$$
\frac{c d_{n}(\lambda, \beta, \delta) \Upsilon_{m}^{l}(n)}{(c+n+1)(1-\delta)} \leq \frac{d_{n}(\lambda, \beta, \gamma) \Upsilon_{m}^{l}(n)}{(1-\gamma)(1-2 \lambda)} .
$$

From (78), we have

$$
\begin{aligned}
\delta \leq( & \left(n^{2}(1+\beta)+n[(\gamma+\beta)+(1+\beta)(1+c \gamma)]\right. \\
+ & (c+1)(\gamma+\beta)+c \beta(1-\gamma)) \\
\times & \left(n^{2}(1+\beta)+n[(\gamma+\beta)+(1+c)(1+\beta)]\right. \\
& \left.+(1+c)(\gamma+\beta)+c(1-\gamma))^{-1}\right)=\Phi(n) .
\end{aligned}
$$

A simple computation will show that $\Phi(n)$ is increasing and $\Phi(n) \geq \Phi(1)$. Using this, the results follow.

\section{Partial Sums}

Silverman [15] determined sharp lower bounds on the real part of the quotients between the normalized starlike or convex functions and their sequences of partial sums. As a natural extension, one is interested in searching results analogous to those of Silverman for meromorphic univalent functions. In this section, motivated essentially by the work of Silverman [15] and Cho and Owa [16], we will investigate the ratio of a function of the form (4) to its sequence of partial sums. Consider

$$
f_{k}(z)=\frac{1}{z-\xi}+\sum_{n=1}^{k} a_{n}(z-\xi)^{n},
$$

when the coefficients are sufficiently small to satisfy the condition analogous to

$$
\sum_{n=1}^{\infty} d_{n}(\lambda, \beta, \gamma) \Upsilon_{m}^{l}(n) a_{n} \leq(1-\gamma)(1-2 \lambda) .
$$

More precisely we will determine sharp lower bounds for $\mathfrak{R}\left(f(z) / f_{k}(z)\right)$ and $\mathfrak{R}\left(f_{k}(z) / f(z)\right)$. In this connection we make use of the well-known results that $\Re((1+w(z)) /(1-$ $w(z)))>0,(z-\xi \in \Delta)$, if and only if $w(z)=\sum_{n=1}^{\infty} c_{n}(z-\xi)^{n}$ satisfies the inequality $|w(z)| \leq|z-\xi|$.

Unless otherwise stated, we will assume that $f$ is of the form (4) and its sequence of partial sums is denoted by (80).

Theorem 17. Let $f(z) \in \mathscr{M}_{m}^{l}(\lambda, \beta, \gamma)$ be given by (4) which satisfies condition (26) and suppose that all of its partial sums (80) do not vanish in $\Delta$. Moreover, suppose that

$$
2-2 \sum_{n=1}^{k}\left|a_{n}\right|-\frac{d_{k+1}(\lambda, \beta, \gamma) \Upsilon_{m}^{l}(k+1)}{(1-\gamma)(1-2 \lambda)} \sum_{n=k+1}^{\infty}\left|a_{n}\right|>0,
$$

$\forall k \in \mathbb{N}$.

Then,

$$
\mathfrak{R}\left(\frac{f(z)}{f_{k}(z)}\right) \geq 1-\frac{(1-\gamma)(1-2 \lambda)}{d_{k+1}(\lambda, \beta, \gamma) \Upsilon_{m}^{l}(k+1)} \quad(z-\xi \in \Delta),
$$


where

$$
\begin{aligned}
& d_{n}(\lambda, \beta, \gamma) \\
& \quad \geq \begin{cases}(1-\gamma)(1-2 \lambda), & \text { if } n=1,2,3, \ldots, k \\
d_{k+1}(\lambda, \beta, \gamma) \Upsilon_{m}^{l}(k+1), & \text { if } n=k+1, k+2, \ldots\end{cases}
\end{aligned}
$$

The result (83) is sharp with the function given by

$$
f(z)=\frac{1}{z-\xi}+\frac{(1-\gamma)(1-2 \lambda)}{d_{k+1}(\lambda, \beta, \gamma) \Upsilon_{m}^{l}(k+1)}(z-\xi)^{k+1} .
$$

Proof. Define the function $w(z)$ by

$$
\begin{aligned}
& w(z) \\
& =\frac{d_{k+1}(\lambda, \beta, \gamma) \Upsilon_{m}^{l}(k+1)}{(1-\gamma)(1-2 \lambda)} \\
& \times\left[\frac{f(z)}{f_{k}(z)}-\left(1-\frac{(1-\gamma)(1-2 \lambda)}{d_{k+1}(\lambda, \beta, \gamma) \Upsilon_{m}^{l}(k+1)}\right)\right] \\
& =1 \\
& +\left(\left(d_{k+1}(\lambda, \beta, \gamma) \Upsilon_{m}^{l}(k+1)\right)\right. \\
& \quad \times((1-\gamma)(1-2 \lambda))^{-1} \\
& \left.\quad \times \sum_{n=k+1}^{\infty} a_{n}(z-\xi)^{n+1}\right) \\
& \left.\times\left(1+\sum_{n=1}^{k} a_{n}(z-\xi)^{n+1}\right)^{-1}\right) .
\end{aligned}
$$

It suffices to show that $\mathfrak{R}(w(z))>0$; hence we find that

$$
\begin{aligned}
& \left|\frac{1+w(z)}{1-w(z)}\right| \\
& \leq\left(\left(d_{k+1}(\lambda, \beta, \gamma) \Upsilon_{m}^{l}(k+1)\right) \times((1-\gamma)(1-2 \lambda))^{-1}\right. \\
& \left.\quad \times \sum_{n=k+1}^{\infty}\left|a_{n}\right|\right) \\
& \times\left(2-2 \sum_{n=1}^{k}\left|a_{n}\right|\right. \\
& \quad-\left(d_{k+1}(\lambda, \beta, \gamma) \Upsilon_{m}^{l}(k+1)\right) \\
& \times((1-\gamma)(1-2 \lambda))^{-1} \\
& \left.\left.\quad \times \sum_{n=k+1}^{\infty}\left|a_{n}\right|\right)^{-1}\right) \leq 1 .
\end{aligned}
$$

From condition (26), it readily yields the assertion (83) of Theorem 17.

To see that the function given by (85) gives the sharp result, we observe that for $z=r e^{i \pi /(k+2)}$

$$
\begin{aligned}
\frac{f(z)}{f_{k}(z)} & =1+\frac{(1-\gamma)(1-2 \lambda)}{d_{k+1}(\lambda, \beta, \gamma) \Upsilon_{m}^{l}(k+1)}(z-\xi)^{n} \\
& \longrightarrow 1-\frac{(1-\gamma)(1-2 \lambda)}{d_{k+1}(\lambda, \beta, \gamma) \Upsilon_{m}^{l}(k+1)},
\end{aligned}
$$

when $r \rightarrow 1^{-}$which shows that the bound (83) is the best possible for each $k \in \mathbb{N}$.

We next determine bounds for $f_{k}(z) / f(z)$.

Theorem 18. Under the assumptions of Theorem 17, we have

$$
\begin{array}{r}
\mathfrak{R}\left(\frac{f_{k}(z)}{f(z)}\right) \geq \frac{d_{k+1}(\lambda, \beta, \gamma) \Upsilon_{m}^{l}(k+1)}{d_{k+1}(\lambda, \beta, \gamma) \Upsilon_{m}^{l}(k+1)+(1-\gamma)(1-2 \lambda)} \\
(z-w \in \Delta),
\end{array}
$$

The result (89) is sharp with the function given by (85).

Proof. By setting

$$
\begin{aligned}
& w(z) \\
& =\left(1+\frac{d_{k+1}(\lambda, \beta, \gamma) \Upsilon_{m}^{l}(k+1)}{(1-\gamma)(1-2 \lambda)}\right) \\
& \times\left[\frac{f_{k}(z)}{f(z)}\right. \\
& \left.\quad-\frac{\left(d_{k+1}(\lambda, \beta, \gamma) \Upsilon_{m}^{l}(k+1) /(1-\gamma)(1-2 \lambda)\right)}{1+\left(d_{k+1}(\lambda, \beta, \gamma) \Upsilon_{m}^{l}(k+1) /(1-\gamma)(1-2 \lambda)\right)}\right]
\end{aligned}
$$

and proceeding as in Theorem 17, we get the desired result and so we omit the details.

Concluding Remark. We observe that, if we specialize the parameters $\lambda$ and $\beta$ as mentioned in Examples 1 and 2, we obtain the analogous results for the classes $\mathscr{M}_{m}^{l}(\beta, \gamma)$ and $\mathscr{M}_{m}^{l}(\gamma)$. Further specializing the parameters $l, m$ various other interesting results (as in Theorems 6-18) can be derived easily for the function class based on interesting differential operators as illustrated below.

(1) For $a_{i}=q^{a_{i}}, b_{j}=q^{b_{j}}, a_{i}>0, b_{j}>0,(i=1, \ldots, l ; j=$ $1, \ldots, m, l=m+1), q \rightarrow 1$, the operator $\mathscr{I}_{m}^{l} f(z)=$ $\mathscr{H}_{m}^{l}\left[a_{1}\right] f(z)$ defined by Liu and Srivastava [10].

(2) For $l=2, m=1, a_{2}=q, q \rightarrow 1$, the operator $\mathscr{L}_{1}^{2}\left[a_{1}, q, b_{1}, q\right] f(z)=\mathscr{L}\left[a_{1} ; b_{1}\right] f(z)$ was introduced and studied by Liu and Srivastava [9].

(3) For $l=1, m=0, a_{1}=\delta+1, q \rightarrow 1$, the operator $\mathscr{L}\left[a_{1} ; b_{1}\right] f(z)=D^{\delta} f(z)=\left(1 / z(1-z)^{\delta+1}\right) * f(z),(\delta>-1)$ 
where $D^{\delta}$ is the differential operator which was introduced by Ganigi and Uralegaddi [17].

\section{Conflict of Interests}

The authors declare that there is no conflict of interests regarding the publication of this paper.

\section{Acknowledgment}

The authors thank the referee for their valuable suggestions.

\section{References}

[1] S. Kanas and F. Ronning, "Uniformaly starlike and convex function and other related classes of univalent functions," Annales Universitatis Mariae Curie-Skłodowska. Sectio A, vol. 53, pp. 95-105, 1991.

[2] A. W. Goodman, "On uniformly starlike functions," Journal of Mathematical Analysis and Applications, vol. 155, no. 2, pp. 364370, 1991.

[3] A. W. Goodman, "On uniformly convex functions," Annales Polonici Mathematici, vol. 56, no. 1, pp. 87-92, 1991.

[4] S. D. Purohit and R. K. Raina, "Certain subclasses of analytic functions associated with fractional q-calculus operators," Mathematica Scandinavica, vol. 109, no. 1, pp. 55-70, 2011.

[5] A. Huda and M. Darus, "Integral operator defined by $q$ analogue of Liu-Srivastava operator," Studia Universitatis BabeşBolyai-Series Mathematica, vol. 58, no. 4, pp. 529-537, 2013.

[6] M. K. Aouf, "On a certain class of meromorphic univalent functions with positive coeffcients," Rendiconti di Matematica e delle sue Applicazioni, vol. 7, no. 11, pp. 209-219, 1991.

[7] M. K. Aouf and G. Murugusundaramoorthy, "On a subclass of uniformly convex functions defined by the Dziok-Srivastava operator," Australian Journal of Mathematical Analysis and Applications, vol. 5, no. 1, article 3, pp. 1-17, 2008.

[8] J. Dziok, G. Murugusundaramoorthy, and J. Sokół, "On certain class of meromorphic functions with positive coeffcients," Acta Mathematica Scientia B, vol. 32, no. 4, pp. 1-16, 2012.

[9] J.-L. Liu and H. M. Srivastava, "Subclasses of meromorphically multivalent functions associated with a certain linear operator," Mathematical and Computer Modelling, vol. 39, no. 1, pp. 35-44, 2004.

[10] J.-L. Liu and H. M. Srivastava, "Classes of meromorphically multivalent functions associated with the generalized hypergeometric function," Mathematical and Computer Modelling, vol. 39, no. 1, pp. 21-34, 2004.

[11] M. L. Mogra, T. R. Reddy, and O. P. Juneja, "Meromorphic univalent functions with positive coeffcients," Bulletin of the Australian Mathematical Society, vol. 32, pp. 161-176, 1985.

[12] S. Owa and N. N. Pascu, "Coeffcient inequalities for certain classes of meromorphically starlike and meromorphically convex functions," Journal of Inequalities in Pure and Applied Mathematics, vol. 4, no. 1, article 17, pp. 1-6, 2003.

[13] C. Pommerenke, "On meromorphic starlike functions," Pacific Journal of Mathematics, vol. 13, pp. 221-235, 1963.

[14] B. A. Uralegaddi and C. Somanatha, "Certain differential operators for meromorphic functions," Houston Journal of Mathematics, vol. 17, no. 2, pp. 279-284, 1991.
[15] H. Silverman, "Partial sums of starlike and convex functions," Journal of Mathematical Analysis and Applications, vol. 209, no. 1, pp. 221-227, 1997.

[16] N. E. Cho and S. Owa, "Partial sums of certain meromorphic functions," Journal of Inequalities in Pure \& Applied Mathematics, vol. 5, no. 2, article 30, 2004.

[17] M. R. Ganigi and B. A. Uralegaddi, "New criteria for meromorphic univalent functions," Bulletin Mathematique de la Societe des Sciences Mathematiques de la Republique Socialiste de Roumanie, vol. 33, no. 1, pp. 9-13, 1989. 


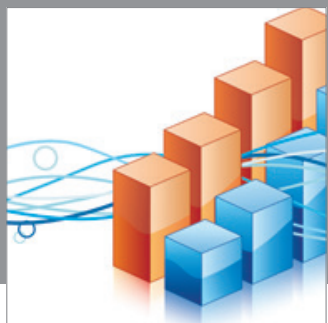

Advances in

Operations Research

mansans

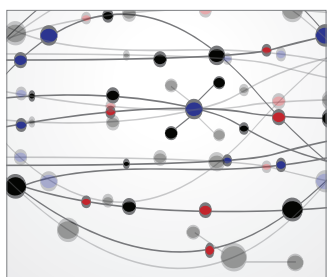

The Scientific World Journal
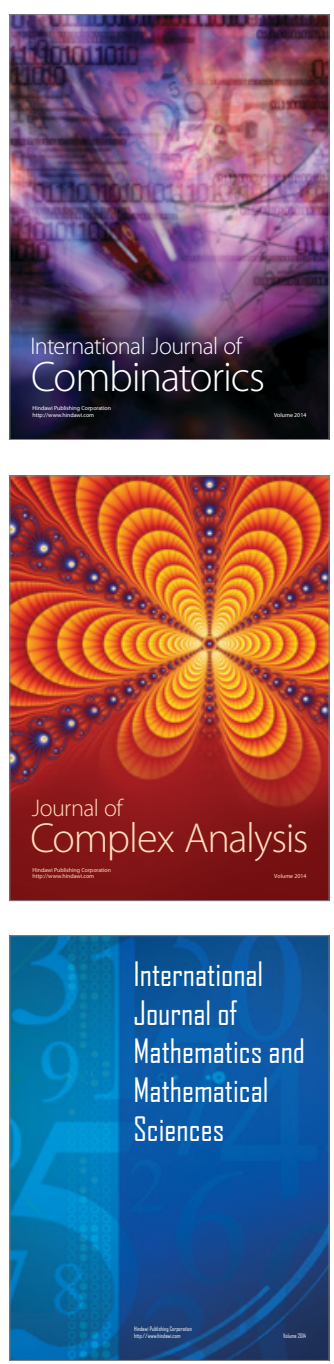
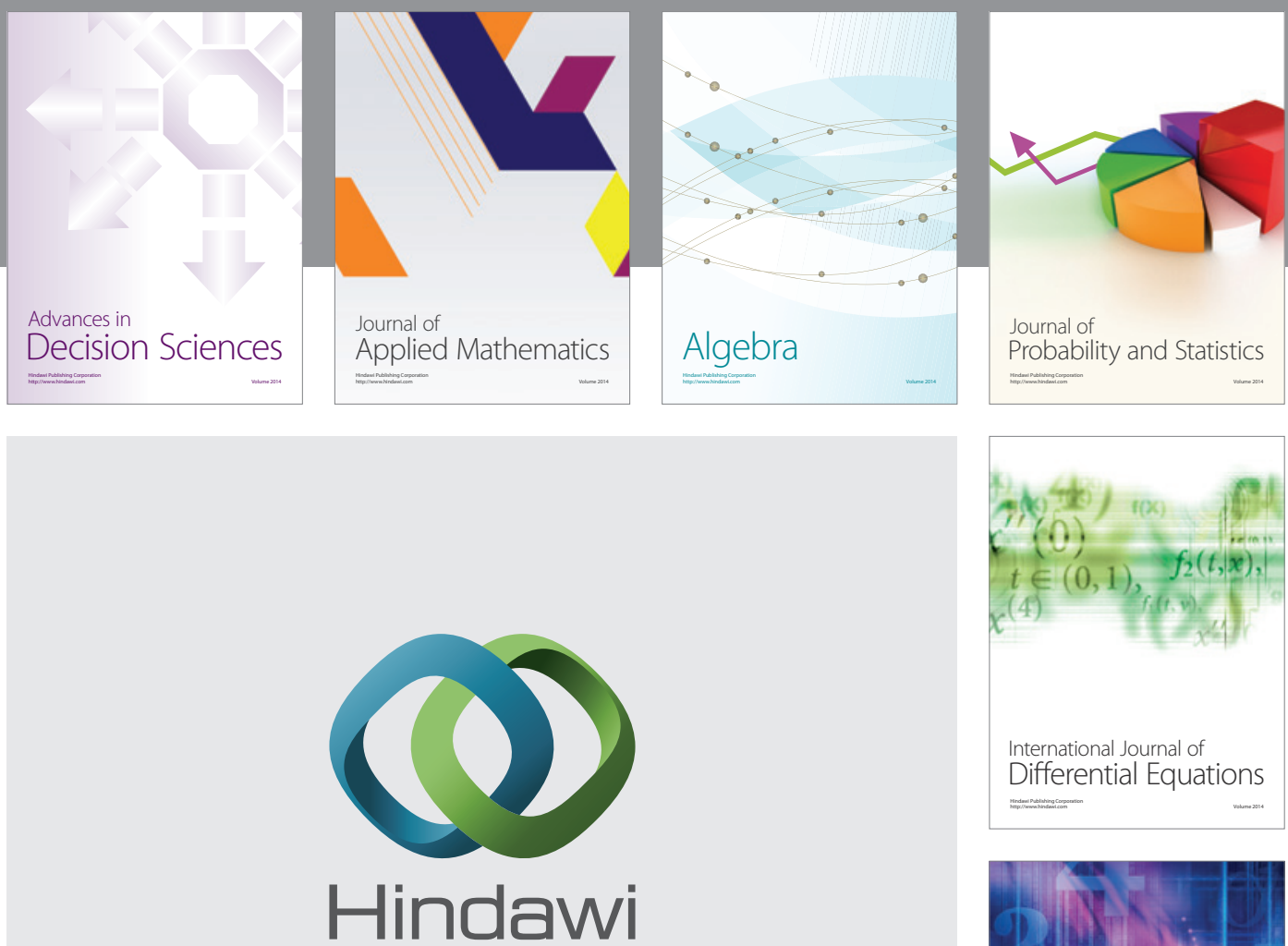

Submit your manuscripts at http://www.hindawi.com
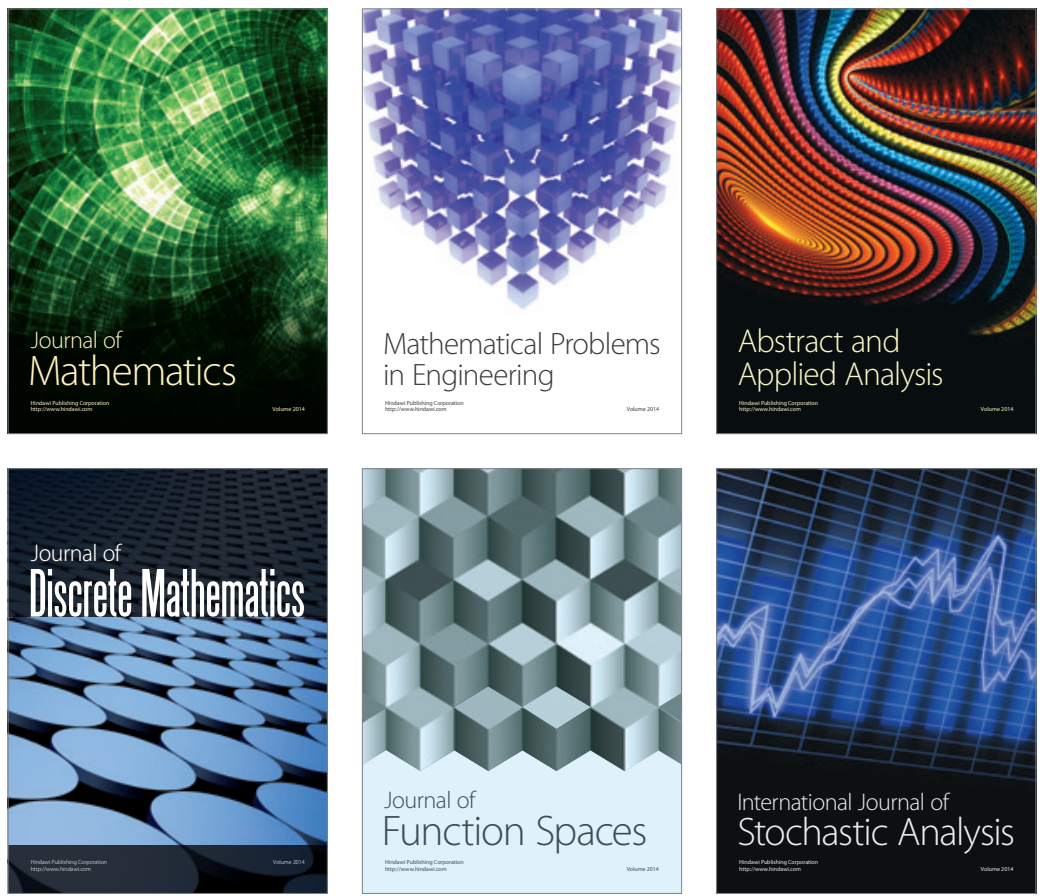

Journal of

Function Spaces

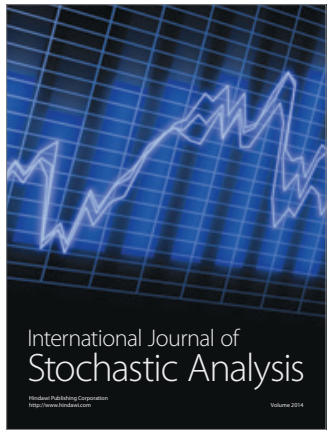

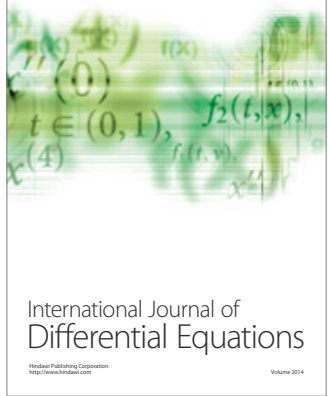
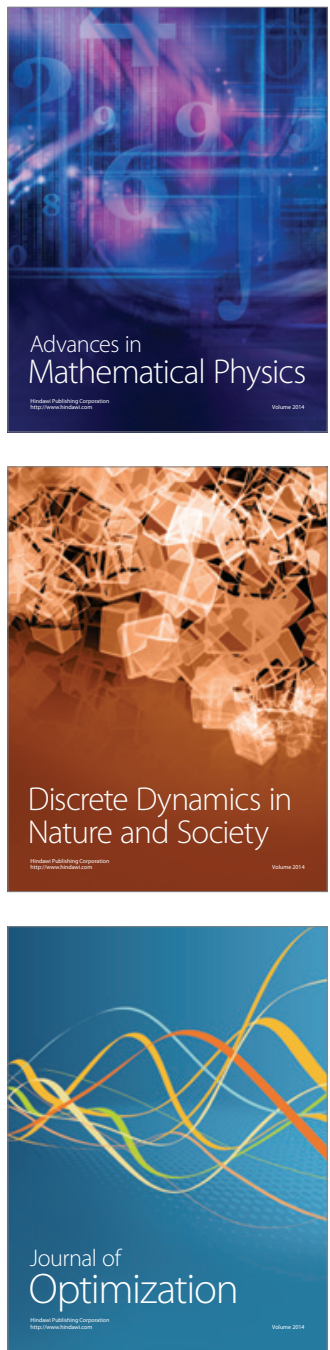